\title{
Adolescentes e adultas jovens e a transmissão materno-infantil do HIV
}

\author{
Adolescents and young adults and mother-to-child transmission of HIV \\ Adolescentes y adultos jóvenes y la transmisión vertical del VIH
}

Recebido: 28/12/2020 | Revisado: 02/01/2021 | Aceito: 06/01/2021 | Publicado: 08/01/2021

\author{
Jefferson do Carmo Dietz \\ ORCID: https://orcid.org/0000-0003-0323-1376 \\ Laboratório Estadual de Saúde Pública Dr. Giovanni Cysneiros, Brasil \\ Hospital Estadual de Doenças Tropicais Dr. Anuar Auad, Brasil \\ E-mail: jeffersondietz@gmail.com \\ Marielly Sousa Borges \\ ORCID: https://orcid.org/0000-0003-0928-9771 \\ Laboratório Estadual de Saúde Pública Dr. Giovanni Cysneiros, Brasil \\ Hospital Estadual de Doenças Tropicais Dr. Anuar Auad, Brasil \\ E-mail: marielly.s.borges@hotmail.com \\ Dayane de Lima Oliveira \\ ORCID: https://orcid.org/0000-0002-6222-9519 \\ Laboratório Estadual de Saúde Pública Dr. Giovanni Cysneiros, Brasil \\ Hospital Estadual de Doenças Tropicais Dr. Anuar Auad, Brasil \\ E-mail: dayane.lima.oliveira@hotmail.com \\ José Geraldo Gomes \\ ORCID: https://orcid.org/0000-0002-5862-9421 \\ Hospital Estadual de Doenças Tropicais Dr. Anuar Auad, Brasil \\ E-mail: jgggyn@gmail.com \\ Edna Joana Cláudio Manrique \\ ORCID: https://orcid.org/0000-0002-8632-3542 \\ Laboratório Estadual de Saúde Pública Dr. Giovanni Cysneiros, Brasil \\ Pontifícia Universidade Católica de Goiás, Brasil \\ E-mail: ednamanrique@gmail.com
}

\begin{abstract}
Resumo
Objetivo: Descrever o perfil de gestantes adolescentes e adultas jovens vivendo com HIV/Aids notificadas em unidade hospitalar de referência em doenças infectocontagiosas do estado de Goiás e analisar as características relacionadas a transmissão materno-infantil do HIV nesta população. Metodologia: Estudo transversal conduzido com dados extraídos de fichas de notificação compulsória de investigação de crianças expostas ao HIV. Foram consideradas as variáveis: idade, município de procedência e escolaridade materna, uso de medicamentos antirretrovirais pela gestante e pelo recém-nascido, tipo de parto (vaginal ou cesáreo) e aleitamento materno. Resultados: Das 157 gestantes vivendo com HIV/Aids, 27,39\% $(n=43)$ eram adolescentes e 72,61\% $(n=114)$ adultas jovens. O perfil desta população aponta para maior procedência de fora da capital goiana, baixa escolaridade e seguimento das recomendações do Ministério da Saúde no quesito utilização de antirretroviral durante a gestação e trabalho de parto. Das crianças expostas ao HIV, 5,09\% $(\mathrm{n}=8)$ infectaram-se por transmissão materno-infantil. A infecção das crianças foi resultado de diferentes combinações da não adoção das medidas preventivas preconizadas pelos protocolas nacionais. Conclusão: No estudo houve predomínio de gestantes adultas jovens de baixa escolaridade vivendo com HIV/Aids. Pertencer ao grupo de gestantes adolescentes ou adultas jovens não influenciou na tomada de medidas profiláticas de transmissão materno-infantil do HIV. Deixar de usar medicamentos antirretrovirais seja no parto ou durante a gestação e fazer o aleitamento materno foram fatores de risco para transmissão materno-infantil do HIV, enquanto o parto cesáreo revelou-se como um fator protetor para maioria dos casos.
\end{abstract}

Palavras-chave: Adolescente; Adulto jovem; HIV; Doenças sexualmente transmissíveis; Transmissão vertical.

\begin{abstract}
Objective: To describe the profile of pregnant adolescents and young adults living with HIV/Aids notified in a reference hospital for infectious diseases in the state of Goiás and analyzing how characteristics related to the motherchild transmission of HIV in this population. Methodology: Cross-sectional study conducted with data extracted from forms of mandatory notification of investigation of children exposed to HIV. Variables were considered: age, municipality of origin and maternal education, use of antiretroviral drugs by the pregnant and the newborn, type of parturition (vaginal or cesarean) and breastfeeding. Results: Of the 157 pregnant living with HIV/Aids, 27,39\% (n = 43) were adolescents and $72,61 \%(n=114)$ young adults. The profile of this population indicate to the largest procedure outside the capital of Goiás, low education level and following the recommendations of the Ministry of Health regarding the use of antiretroviral during pregnancy and parturition. Of the children exposed to HIV, 5,09\% (n $=8$ ) became infected through mother-to-child transmission. The children's infection was the result of different combinations of not adopting the preventive measures recommended by national protocols. Conclusion: There was no
\end{abstract}


study predominantly of young adult pregnant with low education level living with HIV/Aids. Belonging to the group of pregnant adolescents or young adults did not influence the taking of prophylactic measures for mother-to-child transmission of HIV. Do not to use antiretroviral drugs either at parturition or during pregnancy and breastfeeding were risk factors for mother-to-child transmission of HIV, while cesarean parturition proved to be a protective factor for most cases.

Keywords: Adolescent; Young adult; HIV; Sexually transmitted diseases; Vertical transmission.

\begin{abstract}
Resumen
Objetivo: Describir el perfil de las adolescentes y adultas jóvenes embarazadas que viven con VIH/SIDA notificadas en un hospital de referencia por enfermedades infecciosas en el estado de Goiás y analizar las características relacionadas con la transmisión vertical del VIH en esta población. Metodología: Estudio transversal realizado con datos extraídos de formularios de notificación obligatoria de investigación de niños expuestos al VIH. Las variables consideradas fueron: edad, municipio de origen y educación materna, uso de antirretrovirales por la gestante y el recién nacido, tipo de parto (vaginal o cesárea) y amamantamiento. Resultados: De las 157 embarazadas que viven con el VIH/SIDA, 27,39\% ( $n=43)$ eran adolescentes y 72,61\% $(n=114)$ adultas jóvenes. El perfil de esta población apunta a un mayor origen fuera de la capital de Goiás, baja escolaridad y siguiendo las recomendaciones del Ministerio de Salud en cuanto al uso de antirretrovirales durante el embarazo y el parto. De los niños expuestos al $\mathrm{VIH}, 5,09 \%(\mathrm{n}=8)$ se infectaron por transmisión vertical. La infección de los niños fue el resultado de diferentes combinaciones de no adoptar las medidas preventivas recomendadas por los protocolos nacionales. Conclusión: En el estudio hubo predominio de gestantes adultas jóvenes con bajo nivel educativo viviendo con VIH/SIDA. La pertenencia al grupo de adolescentes embarazadas o adultas jóvenes no influyó en la toma de medidas profilácticas para la transmisión del VIH de madre a hijo. La falta de uso de medicamentos antirretrovirales durante el parto o durante el embarazo y el amamantamiento fue un factor de riesgo para la transmisión del VIH de madre a hijo, mientras que el parto por cesárea resultó ser un factor protector en la mayoría de los casos.
\end{abstract}

Palabras clave: Adolescente; Adulto joven; VIH; Enfermedades sexualmente transmisibles; Transmisión vertical.

\title{
1. Introdução
}

A adolescência e o início da vida adulta são marcados por inúmeras mudanças, tanto de natureza física quanto psicológica e social. Neste contexto, indivíduos jovens têm maior propensão para adoção de comportamentos sexuais de risco e consequente vulnerabilidade para contrair infecções sexualmente transmissíveis (IST), a exemplo do vírus da imunodeficiência humana (HIV) e gravidez não planejada (Nery, et al., 2020; Vescovi, Castoldi, Pereira, \& Levandowski, 2016; Vicentim, Queiroz, Sasaki, Santos, \& Souza, 2020). Segundo dados do Ministério da Saúde, a faixa etária de 10 a 24 anos de idade é responsável por mais de 20,0\% das notificações para infecção pelo HIV no período de 2007 a 2020, demonstrando um perfil de juvenização dos casos de HIV (Ministério da Saúde, 2020).

O indivíduo infectado pelo HIV pode evoluir para síndrome da imunodeficiência adquirida (Aids), se não tratado adequadamente, pois o vírus destrói as células de defesa o que aumenta a chance de adoecimento por agentes infecciosos (bactérias, vírus, fungos e protozoários). Ademais, o crescente número de casos em gestantes é preocupante para saúde pública e traz como consequência a possibilidade de transmissão materno-infantil, que pode ocorrer antes do nascimento (ainda no útero), no parto ou na amamentação (Friedrich, Menegotto, Magdaleno, \& Silva, 2016; Ministério da Saúde, 2010). No Brasil quase 90,0\% dos casos de infecção pelo HIV em crianças de até 13 anos de idade são resultado de transmissão materno-infantil (Ministério da Saúde, 2020).

As medidas recomendadas pelo Ministério da Saúde para redução da transmissão materno-infantil do HIV incluem o uso de medicamentos antirretrovirais pela gestante e pelo recém-nascido, o parto cesáreo em casos específicos e a não amamentação (Ministério da Saúde, 2019). O encadeamento destas ações perpassa pela realização adequada do pré-natal e oportunização de testagem para o HIV (Miranda et al., 2016).

Diante do cenário anteriormente exposto, pesquisas relacionadas ao HIV na população de gestantes adolescentes e adultas jovens são necessárias para subsidiar a tomada de decisões para reduzir o impacto desta grave infecção e propiciar o acompanhamento do cumprimento de medidas preventivas de transmissão materno-infantil na realidade local goiana. Nesse sentido o objetivo do trabalho foi escrever o perfil de gestantes adolescentes e adultas jovens vivendo com HIV/Aids, bem 
como verificar se houve diferença na adoção de medidas preventivas para a transmissão materno-infantil do HIV entre o grupo de adolescentes e o de adultas jovens e a chance de soroconversão das crianças expostas relacionada a cada medida preventiva, de casos notificados em unidade hospitalar terciária de referência em doenças infectocontagiosas do estado de Goiás.

\section{Metodologia}

Estudo transversal conduzido com dados extraídos das fichas de notificação compulsória de investigação de crianças expostas ao HIV arquivadas pelo Núcleo Hospitalar de Vigilância Epidemiológica (NHVE) de uma unidade goiana de referência em doenças infectocontagiosas.

Para inclusão no estudo foram contemplados dados provenientes de casos com mães vivendo com HIV/Aids na faixa etária de 14 a 24 anos com a investigação encerrada no período de 2010 a 2016. É importante destacar que para considerar a investigação encerrada ocorre o seguimento das crianças expostas ao HIV até os 18 meses de idade e a confirmação realizada por meio de evidência laboratorial da infecção pelo HIV. Assim, em crianças menores de 18 meses de idade expostas ao HIV por transmissão materno-infantil, foi considerada infectada quando houve a presença de RNA viral plasmático detectável acima de 10.000 cópias/mL ou detecção do DNA pró-viral em duas amostras obtidas em momentos diferentes. Para as crianças com 18 meses de idade ou mais e expostas ao HIV por transmissão materno-infantil, foram consideradas infectadas pelo HIV quando uma amostra de soro foi positiva em um teste de triagem ou um confirmatório para pesquisa de anticorpos anti-HIV ou dois testes rápidos. A adoção destes parâmetros obedeceu ao fluxograma do consenso sobre terapia antirretroviral para crianças infectadas pelo HIV em vigência no período do estudo (Ministério da Saúde, 2009).

Para categorizar as gestantes como pertencentes ao grupo de adolescentes ou adultas jovens adotou-se adaptação de conceito da Organização Mundial da Saúde (OMS) e Ministério da Saúde que considera indivíduos com idade entre 10 e 20 anos incompletos como adolescentes e entre 15 e 24 anos como jovens (Ministério da Saúde, 2013). Dado que há um intervalo etário comum a adolescência e juventude, o grupo de pesquisadores optou por delimitar a adolescência entre 14 e 19 anos de idade (uma vez que não foram identificadas gestantes com idade inferior) e nomear o grupo etário de 20 a 24 anos de adulto jovem.

As variáveis maternas consideradas foram: idade (em anos), procedência (município), escolaridade, uso de antirretroviral durante a gestação, uso de antirretroviral durante o parto e evolução da gestação (tipo de parto). Já as variáveis alvo de análise relacionadas à caracterização da transmissão materno-infantil do HIV foram: uso de antirretroviral durante a gestação, uso de antirretroviral durante o parto, tipo de parto (vaginal ou cesáreo), uso de antirretroviral oral pela criança exposta ao HIV e aleitamento materno. As informações foram inseridas em um banco de dados eletrônico do Microsoft ${ }^{\circledR}$ Office Excel para posterior tabulação e análise e os resultados expressos em frequências absolutas e relativas. Para o cálculo da taxa de transmissão materno-infantil do HIV na amostra estudada dividiu-se o número de crianças infectadas pelo número de crianças expostas e o resultado multiplicado por cem.

O teste estatístico do Qui-quadrado com correção de Yates foi utilizado para verificar se houve diferença estatística ente os dois grupos (adolescentes e adultas jovens) com relação às medidas preventivas preconizadas pelo Ministério da Saúde quanto a transmissão materno-infantil do HIV, demostrando significância com o valor $p<0,05$. O teste de Odds ratio (OR) com seus respectivos intervalos de confiança de 95\% (IC95\%) foi empregado para mensurar a chance de transmissão maternoinfantil no que tange a cada medida preventiva. Todos os testes foram calculados por meio do programa estatístico BioEstat 5.3 .

O presente estudo foi aprovado por Comitê de Ética em Pesquisa de hospital referência em doenças infectocontagiosas do estado de Goiás sob parecer de nº 3.374.603 (CAAE 14119619.1.0000.0034), conforme recomendações da Resolução nº 466 do Conselho Nacional de Saúde, de 12 de dezembro de 2012. 


\section{Resultados}

No período de estudo foram identificadas 157 gestantes adolescentes e adultas jovens vivendo com HIV/Aids. A Tabela 1 apresenta o perfil destas gestantes, onde 72,61\% ( $\mathrm{n}=114)$ eram adultas jovens e 27,39\% $(\mathrm{n}=43)$ adolescentes. Do total, $74,52 \%$ ( $\mathrm{n}=117$ ) era procedente de fora da capital goiana. A escolaridade quando informada aponta para menor grau de instrução, com predominância de casos entre gestantes com ensino fundamental completo 13,38\% e incompleto 8,28\%. No quesito utilização de antirretroviral durante a gestação e trabalho de parto, grande parcela seguiu o recomendado pelo Ministério da Saúde, perfazendo 81,53\% (n=128) e 62,42\% (n=98), respectivamente. Já com relação a evolução da gravidez, $66,88 \%(n=105)$ resultaram em parto cesáreo.

Tabela 1 - Perfil das gestantes adolescentes e adultas jovens vivendo com HIV/Aids.

\begin{tabular}{|c|c|c|}
\hline Variáveis & $\mathbf{N}=157$ & $\%$ \\
\hline \multicolumn{3}{|l|}{ Faixa etária (anos) } \\
\hline $14-19$ & 43 & 27,39 \\
\hline $20-24$ & 114 & 72,61 \\
\hline \multicolumn{3}{|l|}{ Procedência } \\
\hline Goiânia & 40 & 25,48 \\
\hline Outros municípios & 117 & 74,52 \\
\hline \multicolumn{3}{|l|}{ Escolaridade } \\
\hline Analfabeto & 3 & 1,91 \\
\hline Ensino fundamental incompleto & 13 & 8,28 \\
\hline Ensino fundamental completo & 21 & 13,38 \\
\hline Ensino médio incompleto & 5 & 3,18 \\
\hline Ensino médio completo & 13 & 8,28 \\
\hline Ensino superior incompleto & 1 & 0,64 \\
\hline Ensino superior completo & 1 & 0,64 \\
\hline Ignorado & 100 & 63,69 \\
\hline \multicolumn{3}{|c|}{ Uso de antirretroviral durante a gestação } \\
\hline Sim & 128 & 81,53 \\
\hline Não & 26 & 16,56 \\
\hline Ignorado & 3 & 1,91 \\
\hline \multicolumn{3}{|c|}{ Uso de antirretroviral durante o parto } \\
\hline $\operatorname{Sim}$ & 98 & 62,42 \\
\hline Não & 36 & 22,93 \\
\hline Ignorado & 23 & 14,65 \\
\hline \multicolumn{3}{|l|}{ Evolução da gravidez } \\
\hline Parto vaginal & 51 & 32,48 \\
\hline Parto cesáreo & 105 & 66,88 \\
\hline Ignorado & 1 & 0,64 \\
\hline
\end{tabular}


Fonte: Autores.

A Tabela 2 demonstra que não houve diferença estatisticamente significante entre o grupo de adolescentes e o grupo de adultas jovens quanto a tomada de medidas preventivas de transmissão materno-infantil do HIV.

Tabela 2 - Tomada de medidas preventivas de transmissão materno-infantil do HIV por adolescentes e adultas jovens.

\begin{tabular}{|c|c|c|c|c|}
\hline Variáveis & $\begin{array}{l}\text { Adolescentes } \\
\qquad(\mathrm{n}=43)\end{array}$ & $\begin{array}{l}\text { Adultas jovens } \\
\qquad(\mathrm{n}=114)\end{array}$ & $\chi^{2}$ & p-valor \\
\hline \multicolumn{5}{|c|}{ Uso de antirretroviral durante a gestação } \\
\hline Sim & 35 & 93 & \multirow{2}{*}{0,04} & \multirow{2}{*}{0,84} \\
\hline Não & 7 & 19 & & \\
\hline Ignorado & 1 & 2 & - & - \\
\hline \multicolumn{5}{|c|}{ Uso de antirretroviral durante o parto } \\
\hline Sim & 27 & 71 & \multirow{2}{*}{0,04} & \multirow{2}{*}{0,84} \\
\hline Não & 10 & 26 & & \\
\hline Ignorado & 6 & 17 & - & - \\
\hline \multicolumn{5}{|c|}{ Evolução da gravidez } \\
\hline Parto cesáreo & 31 & 74 & \multirow{2}{*}{0,35} & \multirow{2}{*}{0,55} \\
\hline Parto vaginal & 12 & 39 & & \\
\hline Ignorado & - & 1 & - & - \\
\hline \multicolumn{5}{|c|}{ Aleitamento materno } \\
\hline $\operatorname{Sim}$ & 4 & 7 & \multirow[t]{2}{*}{0,08} & \multirow{2}{*}{0,77} \\
\hline Não & 37 & 98 & & \\
\hline Ignorado & 2 & 9 & - & - \\
\hline
\end{tabular}

$\chi^{2}$ - Qui-quadrado com correção de Yates. Para o cálculo do Qui-quadrado foram excluídos os dados ignorados. Fonte: Autores.

Neste estudo, como apresentado na Tabela 3, constituíram-se fatores de risco para transmissão materno-infantil do HIV o não uso de antirretroviral durante a gestação (OR 3,20; IC95\% 0,71-14,36) e parto (OR: 4,36; IC95\% 0,70-27,27) e fazer o aleitamento materno (OR 1,83; IC95\% 0,20-16,37), enquanto o parto cesáreo (OR: 0,67; IC95\% 0,13-3,46) foi um fator protetor. Não usar antirretroviral durante a gestação resultou em chance 3,2 vezes maior de transmissão do HIV, já a não utilização durante o parto aumenta a chance de transmissão em 4,36 vezes. A possibilidade de infecção da criança exposta ao HIV foi 1,83 vezes maior em casos onde houve aleitamento materno e o risco 0,67 vezes menor quando realizado o parto cesáreo. 
Tabela 3 - Análise do desfecho das crianças expostas ao HIV e medidas preventivas de transmissão materno-infantil do HIV.

\begin{tabular}{|c|c|c|c|}
\hline \multirow{2}{*}{ Variáveis } & \multicolumn{2}{|c|}{ Criança infectada } & \multirow{2}{*}{ OR (IC95\%) } \\
\hline & Sim & Não & \\
\hline Uso de antirretroviral durante a gestação & \multicolumn{2}{|c|}{$(n=154)$} & \\
\hline Sim & 5 & 123 & 3,20 \\
\hline Não & 3 & 23 & $(0,71-14,36)$ \\
\hline Uso de antirretroviral durante o parto & \multicolumn{2}{|c|}{$(n=134)$} & \\
\hline Sim & 2 & 96 & 4,36 \\
\hline Não & 3 & 33 & $(0,70-27,27)$ \\
\hline Evolução da gravidez & \multicolumn{2}{|c|}{$(n=156)$} & \\
\hline Parto cesáreo & 6 & 99 & 0,67 \\
\hline Parto vaginal & 2 & 49 & $(0,13-3,46)$ \\
\hline Aleitamento materno & \multicolumn{2}{|c|}{$(n=146)$} & \\
\hline Sim & 1 & 10 & 1,83 \\
\hline Não & 7 & 128 & $(0,20-16,37)$ \\
\hline
\end{tabular}

Fonte: Autores.

Do total de crianças expostas, 5,09\% $(\mathrm{n}=8)$ infectaram-se com HIV por transmissão materno-infantil. Como evidenciado na Tabela 4, as crianças expostas e infectadas não receberam todas as medidas preventivas previstas. As crianças C1 e C7 nasceram de parto vaginal e as mães não fizeram uso de antirretroviral durante a gestação. O uso de antirretroviral durante o parto não foi informado para as crianças C2, C5 e C6 e não realizado para C1 e C3. As crianças C4 e C7 não completaram o esquema de uso de antirretroviral oral. Na criança C8 observou-se ausência de prevenção para transmissão materno-infantil do HIV, havendo inclusive aleitamento materno.

Tabela 4 - Características dos casos de crianças infectadas pelo HIV por transmissão materno-infantil.

\begin{tabular}{|c|c|c|c|c|c|c|c|c|}
\hline & C1 & $\mathrm{C} 2$ & $\mathrm{C3}$ & $\mathrm{C} 4$ & $\mathrm{C5}$ & C6 & C7 & $\mathrm{C8}$ \\
\hline $\begin{array}{l}\text { Mãe fez uso de antirretroviral } \\
\text { durante a gestação }\end{array}$ & Não & Sim & Sim & Sim & Sim & Sim & Não & Não \\
\hline Tipo de parto & PV & $\mathrm{PC}$ & PC & $\mathrm{PC}$ & $\mathrm{PC}$ & $\mathrm{PC}$ & PV & $\mathrm{PC}$ \\
\hline $\begin{array}{l}\text { Uso de antirretroviral durante o } \\
\text { parto }\end{array}$ & Não & Ign & Não & Sim & $\operatorname{Ign}$ & Ign & Sim & Não \\
\hline $\begin{array}{l}\text { Uso de profilaxia antirretroviral } \\
\text { oral }\end{array}$ & Sim & Sim & Sim & Sim & Não & Sim & Sim & Não \\
\hline $\begin{array}{l}\text { Tempo de uso de profilaxia } \\
\text { antirretroviral oral (semanas) }\end{array}$ & $\operatorname{Ign}$ & 6 & 6 & 3 a 5 & 0 & 6 & 3 a 5 & 0 \\
\hline Aleitamento materno & Não & Não & Não & Não & Não & Não & Não & Sim \\
\hline
\end{tabular}

$\mathrm{C}=$ criança $. \mathrm{PV}=$ parto vaginal $. \mathrm{PC}=$ parto cesáreo. Ign $=$ Ignorado. Fonte: Autores. 


\section{Discussão}

Adultas jovens constituíram 72,61\% $(\mathrm{n}=114)$ da amostra do presente estudo. No cenário nacional brasileiro, desde o ano 2000, a maior proporção de casos notificados de gestantes infectadas pelo HIV ocorre justamente na faixa etária entre 20 e 24 anos (Ministério da Saúde, 2020). O comportamento sexual e o baixo poder socioeconômico da população de adolescentes e jovens são apontados como fatores que contribuem para vulnerabilidade do grupo para disseminação do HIV e de outras IST (Costa et al., 2011). Considerando os adolescentes em especial, a literatura também indica baixa preocupação destes com medidas preventivas não em decorrência da desinformação, mas sim do comportamento próprio da idade (Cruz et al., 2018). Apesar disso, o presente estudo não evidenciou diferença com relação a adoção de medidas preventivas de transmissão materno-infantil entre o grupo de gestantes adolescentes e gestantes adultas jovens.

A maioria da população de gestantes da presente pesquisa era procedente de fora da capital goiana. Essa constatação é concordante com pesquisas que demonstram a descentralização da epidemia de HIV e tendência de aumento em municípios de pequeno, médio e grande porte. Este fenômeno pode refletir falhas na organização do sistema de saúde local e dificultar o atendimento desta população (Menezes, Palacios, Peixoto, Alcântara, \& Bichara, 2013).

Sobre a escolaridade demostrou-se baixo grau de instrução, com predominância de casos entre gestantes com ensino fundamental completo ou incompleto. Este resultado é concordante com outras pesquisas sobre a temática (Bick, Ferreira, Sampaio, Padoin, \& Paula, 2018; Oliveira, Lima, Verde, Holanda, Sousa, \& Andrade, 2020). Segundo a literatura, a pouca instrução formal aumenta a proporção de oportunidades perdidas para a realização da triagem sorológica para HIV e outras IST (Costa et al., 2011), entretanto, é válido destacar que a escolaridade na população do presente estudo decorre da pouca idade. Outro fator que contribui para esta realidade é o abandono escolar, frequente após gestações na adolescência (Costa et al., 2011; Vicentim et al., 2020).

Grande parcela da amostra do estudo realizou tratamento com medicação antirretroviral durante a gestação. Este fato é justificado na literatura pela preocupação da mulher em infectar o filho (Pinto, Maia, Pereira, \& Levandowski, 2017), sendo que três quartos da população geral de gestantes adotam esta medida para prevenção da transmissão do HIV para sua prole (Friedrich et al., 2016). Neste estudo a maioria das mães cujos filhos apresentaram soroconversão referiram o uso de antirretrovirais quando gestantes, porém presume-se que isso não assegure a adequada adesão ao tratamento, uma vez que o instrumento de coleta de dados oferecia como possibilidades de resposta apenas afirmação ou negação. Atualmente a recomendação é realizar o monitoramento da carga viral em gestantes vivendo com HIV/Aids, ação que contribui para definição da via de parto e para identificar chance de transmissão para a criança exposta em casos de tratamento irregular ou ineficiente (Ministério da Saúde, 2019).

O Brasil é referência mundial em políticas públicas de profilaxia da transmissão materno-infantil do HIV (Bick et al., 2018). O protocolo nacional vigente no período do estudo preconizava o uso de antirretroviral durante a gestação e trabalho de parto, a realização de cesárea eletiva, o uso de antirretroviral oral pelo recém-nascido até a $6^{\text {a }}$ semana de vida e a não amamentação (Ministério da Saúde, 2010). A orientação atual para duração do tratamento do neonato é de 4 semanas e recomenda-se a realização de cesárea eletiva para mulheres com carga viral desconhecida no momento do parto ou carga viral maior que 1.000 cópias $/ \mathrm{mL}$ até a $34^{\mathrm{a}}$ semana de gestação (Ministério da Saúde, 2019). Os fatores associados a maior risco ou proteção da transmissão materno-infantil do HIV aqui demonstrados corroboram com a literatura sobre a temática (Bick et al., 2018; Miranda et al., 2016). Não por acaso, as orientações do Ministério da Saúde contemplam estas situações.

A taxa geral de transmissão materno-infantil do HIV estimada no período desta pesquisa foi de 5,09\%. Ao considerar que na ausência de intervenções preventivas a infecção ocorre em até 31,0\% das crianças expostas, a taxa observada comprova a efetividade da aplicação das medidas de prevenção preconizadas na redução da transmissão. Porém, levando em conta que a adoção de todas as intervenções terapêuticas resulta em taxas de transmissão inferiores a 2,0\% (Friedrich et al., 2016), o 
número de 5,09\% evidencia também oportunidades perdidas de prevenção.

No presente estudo a soroconversão de crianças expostas ao HIV ocorreu em diferentes cenários de não adoção das medidas previstas pelo Ministério da Saúde. As crianças C1 e C7 nasceram de parto vaginal e as mães não fizeram uso de antirretroviral durante a gestação. Estas ocorrências traduzem lacunas na assistência à saúde materna e durante o trabalho de parto. No Brasil, estudo demonstra que 72,0\% dos diagnósticos de infecção pelo HIV entre gestantes adolescentes e adultas jovens só ocorrem durante a triagem pré-natal (Lima, Reis, Cardoso, \& Stefani, 2016). A assistência pré-natal inadequada resulta na perda de oportunidade para triagem sorológica para HIV e outras IST. Além disso, a cobertura de testagem para HIV aumenta com a idade e a escolaridade (Pereira et al., 2016), ambas baixas na amostra deste estudo.

O tratamento da infecção materna tem o objetivo de reduzir a quantidade de vírus circulante no sangue e consequentemente prevenir a contaminação da criança ao entrar em contato com secreções da mãe no momento do parto. Cerca de $65,0 \%$ das infecções ocorrem justamente no momento do nascimento e a supressão da carga viral a níveis indetectáveis reduz a possibilidade de transmissão materno-infantil para taxas inferiores a 1,0\% (Friedrich et al., 2016). Em adição a pouca idade, a adesão ao tratamento pode ser impactada pela vivência simultânea da gravidez/maternidade e infecção pelo HIV (Vescovi et al., 2016).

O uso de antirretroviral durante o trabalho de parto não foi informado para as crianças C2, C5 e C6 e não realizado para C1, C3 e C8. Essa medida previne até 90,0\% dos casos de transmissão materno-infantil (Ministério da Saúde, 2010). As crianças C4 e C7 não completaram o esquema de uso de antirretroviral oral até a $6^{\text {a }}$ semana de vida, conduta que visa inibir a replicação e a disseminação viral (Friedrich et al., 2016).

O aleitamento materno é responsável por um terço à metade da transmissão materno-infantil do HIV (Friedrich et al., 2016). O mesmo deve ser substituído por fórmula láctea infantil, sendo contraindicado o aleitamento misto ou cruzado (amamentação da criança por outra nutriz). Para assegurar a tomada desta medida o Ministério da Saúde oferece a criança exposta o direito de receber a fórmula infantil gratuitamente pelo menos até o sexto mês de vida (Ministério da Saúde, 2014). Entretanto limitações sociais, culturais e econômicas podem interferir na adesão a alimentação substitutiva. O desejo materno de amamentar e o simbolismo social em torno desta prática são exemplos de dificultadores. Além disso, fragilidades no sistema de saúde resultam na falta de informação sobre a gratuidade da fórmula artificial e de orientação profissional (Alvarenga et al., 2019; Pinto et al., 2017).

Como explicitado, o conjunto de estratégias para prevenção da transmissão materno-infantil do período do estudo não foi seguido em sua plenitude. Informações provenientes de fichas de notificação estão sujeitos a limitação devido ao grande percentual de incompletude de dados, entretanto, avaliar os fatores que culminam na soroconversão de crianças expostas ao HIV cujas mães são adolescentes e jovens é de suma importância visto que esta situação ocorre mesmo com as medidas delimitadas e os protocolos disponíveis. Com isso, o modelo de abordagem e cuidado pode ser mais eficiente com enfoque nas características do grupo populacional aqui descritas.

\section{Conclusão}

No presente estudo houve predomínio de gestantes adultas jovens de baixa escolaridade vivendo com HIV/Aids. A maioria das mães adolescentes e adultas jovens eram procedentes de fora da capital goiana, realizaram parto cesáreo e seguiram as recomendações de uso de medicação antirretroviral durante a gestação e trabalho de parto. Pertencer ao grupo de gestantes adolescentes ou adultas jovens não influenciou na tomada de medidas profiláticas de transmissão materno-infantil do HIV. Deixar de usar medicamentos antirretrovirais seja no parto ou durante a gestação e fazer o aleitamento materno foram fatores de risco para transmissão materno-infantil do HIV, enquanto o parto cesáreo demonstrou-se como um fator protetor quando considerada a totalidade de casos. Os casos de soroconversão de crianças expostas ao HIV foram resultado de 
diferentes associações de ausência da tomada de medidas preventivas recomendadas pelos protocolos nacionais. Os resultados expostos reforçam a necessidade de intensificação de medidas de atenção à população adolescente e adulta jovem para reduzir a transmissão materno-infantil.

\section{Referências}

Alvarenga, W. A., Nascimento, L. C., Lima, C. L., Fabbro, M. R. C., Bussadori, J. C. C., Melo, S. S. S., Cartagena-Ramos, D., \& Dupas, G. (2019). Mães vivendo com HIV: a substituição do aleitamento por fórmula láctea infantil. Revista Brasileira de Enfermagem, 72(5), 1153-1160.

Bick, M. A., Ferreira, T., Sampaio, C. D. O., Padoin, S. M. D. M., \& Paula, C. C. D. (2018). Perfil de gestantes infectadas e crianças expostas ao HIV atendidas em serviço especializado do sul do Brasil. Revista Brasileira de Saúde Materno Infantil, 18(4), $791-801$.

Costa, M. C. O., Santos, B. C., Souza, K. D., Cruz, N. L. D. A., Santana, M. C., \& Nascimento, O. D. (2011). HIV/AIDS e sífilis entre gestantes adolescentes e adultas jovens: fatores de exposição e risco dos atendimentos de um programa de DST/HIV/AIDS na rede pública de saúde/SUS, Bahia, Brasil. Revista Baiana de Saúde Pública, 35(1), 179-185.

Cruz, L. Z., Andrade, M. S., Paixão, G. P. N., Silva, R. S., Maciel, K. M. N., \& Fraga, C. D. S. (2018). Conhecimento dos adolescentes sobre contracepção e infecções sexualmente transmissíveis. Revista Adolescência \& Saúde, 15(2), 7-18.

Friedrich, L., Menegotto, M., Magdaleno, A. M., \& Silva, C. D. (2016). Transmissão vertical do HIV: uma revisão sobre o tema. Boletim Científico de Pediatria, 5(3), 81-86.

Lima, Y. A. R., Reis, M. N. G., Cardoso, L. P. V., \& Stefani, M. M. A. (2016). HIV-1 infection and pregnancy in young women in Brazil: socioeconomic and drug resistance profiles in a cross-sectional study. BMJ Open, 6(7), e010837.

Menezes, L. D. S. H. D., Palacios, V. R. D. C. M., Peixoto, C. A. D. S., Alcântara, M. S. D. V., \& Bichara, C. N. C. (2013). Perfil epidemiológico de grávidas HIV positivas atendidas em maternidade pública de referência. Revista Paraense de Medicina, 27(2), 57-64.

Ministério da Saúde. (2009). Recomendações para terapia antirretroviral em crianças e adolescentes infectados pelo HIV: manual de bolso. Secretaria de Vigilância em Saúde, Programa Nacional de DST e Aids. http://www.aids.gov.br/system/tdf/pub/2016/59203/consenso_pediatrico.pdf?file= $1 \&$ type $=$ node $\&$ id $=59203 \&$ force $=1$

Ministério da Saúde. (2010). Recomendações para profilaxia da transmissão vertical do HIV e terapia antirretroviral em gestantes: manual de bolso. Secretaria de Vigilância em Saúde, Programa Nacional de DST e Aids. http://www.aids.gov.br/system/tdf/pub/2016/59199/consenso_ gestantes_2010_vf.pdf?file=1\&type=node\&id=59199\&force=1

Ministério da Saúde. (2013). Recomendações para a atenção integral a adolescentes e jovens vivendo com HIV-Aids. Secretaria de Vigilância em Saúde, Departamento de DST, Aids e Hepatites Virais. http://www.aids.gov.br/system/tdf/pub/2016/59198/adolescentes_07032013_web_pdf_ 20485.pdf? file $=1 \&$ type $=$ node $\&$ id $=59198 \&$ force $=1$

Ministério da Saúde. (2014). Protocolo clínico e diretrizes terapêuticas para manejo da infecção pelo HIV em crianças e adolescentes. Secretaria de Vigilância em Saúde, Departamento de DST, Aids e Hepatites Virais.

Ministério da Saúde. (2019). Protocolo clínico e diretrizes terapêuticas para prevenção da transmissão vertical de HIV, sífilis e hepatites virais. Secretaria de Vigilância em Saúde, Departamento de Doenças de Condições Crônicas e Infecções Sexualmente Transmissíveis. http://www.aids.gov.br/system/ tdf/pub/2016/57801/miolo_pcdt_tv_08_2019.pdf?file=1\&type=node\&id=57801\&force=1

Ministério da Saúde. (2020). Boletim Epidemiológico HIV/Aids 2020. Secretaria de Vigilância em Saúde, Departamento de Doenças de Condições Crônicas e Infecções Sexualmente Transmissíveis. http://www.aids.gov.br/system/tdf/pub/2016/67456/boletim_hiv_aids_2020_com_marcas_2.pdf?file=1\&type= node $\&$ id $=67456 \&$ force $=1$

Miranda, A. E., Pereira, G. F. M., Araújo, M. A. L., Silveira, M. F. D., Tavares, L. D. L., Silva, L. C. F. D., Moreira-Silva, S. F., \& Saraceni, V. (2016). Avaliação da cascata de cuidado na prevenção da transmissão vertical do HIV no Brasil. Cadernos de Saúde Pública, $32(9)$, e00118215.

Nery, C. L. P. D., Teixeira, C. S. S., Santos, D. S. S., Silva, J. S., Conceição, M. M., \& Felzemburgh, R. D. M. (2020). Fatores associados à gravidez em adolescentes escolares no Brasil. Revista Adolescência \& Saúde, 17(2), 07-16.

Oliveira, E. H., Lima, H. R., Verde, R. M. C. L., Holanda, E. C., Sousa, F. D. C. A., \& Andrade, S. M. (2020). HIV e gestação em adolescentes e adultas jovens: perfil epidemiológico e fatores associados à transmissão. Research, Society and Development, 9(8), e702986536-e702986536.

Pereira, G. F. M., Sabidó, M., Caruso, A., Oliveira, S. B. D., Mesquita, F., \& Benzaken, A. S. (2016). HIV prevalence among pregnant women in Brazil: a national survey. Revista Brasileira de Ginecologia e Obstetrícia, 38(8), 391-398.

Pinto, M. D., Maia, G. N., Pereira, M., \& Levandowski, D. C. (2017). Mães adolescentes que vivem com o HIV: uma investigação qualitativa sobre a "Constelação da Maternidade". Psicologia Clínica, 29(3), 381-401.

Vescovi, G., Castoldi, L., Pereira, M. D., \& Levandowski, D. C. (2016). Fatores de risco para a maternidade entre adolescentes vivendo com HIV. Psicologia em Estudo, 21(1), 41-52.

Vicentim, A. L., Queiroz, A. M. A., Sasaki, N. S. G. M. S., Santos, M. L. S. G., \& de Souza, L. H. (2020). Influência de variáveis socioeconômicas, demográficas e de comportamento sexual no abandono escolar de gestantes adolescentes. Research, Society and Development, 9(11), e3399119968e3399119968. 